\section{BRAZIULIAN JOURNAL \\ OFMEDICAL AND BIOLOGICAL RLSF.ARCH}

www.bjournal.com.br
ISSN 0100-879X

Volume 42 (11) 993-1118 November 2009

BIOMEDICAL SCIENCES

AND

CLINICAL INVESTIGATION

Braz J Med Biol Res, November 2009, Volume 42(11)1110-1112

Comparison of I-FISH and G-banding for the detection of chromosomal abnormalities during the evolution of myelodysplastic syndrome

R.F. Pinheiro and M.L.L.F. Chauffaille

The Brazilian Journal of Medical and Biological Research is partially financed by
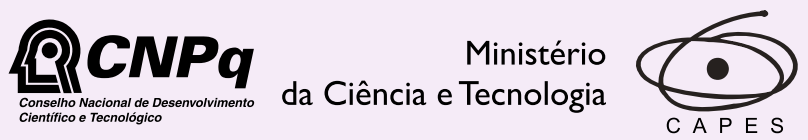

Ministério da Educação

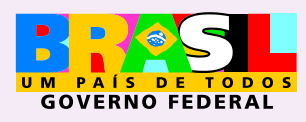

Institutional Sponsors

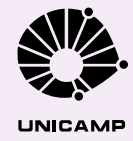




\title{
Comparison of I-FISH and G-banding for the detection of chromosomal abnormalities during the evolution of myelodysplastic syndrome
}

\author{
R.F. Pinheiro and M.L.L.F. Chauffaille \\ Disciplina de Hematologia e Hemoterapia, Escola Paulista de Medicina, \\ Universidade Federal de São Paulo, São Paulo, SP, Brasil
}

\begin{abstract}
Myelodysplastic syndrome (MDS) patients with a normal karyotype constitute a heterogeneous group from a biological standpoint and their outcome is often unpredictable. Interphase fluorescence in situ hybridization (I-FISH) studies could increase the rate of detection of abnormalities, but previous reports in the literature have been contradictory. We performed I-FISH and conventional karyotyping (G-banding) on 50 MDS patients at diagnosis, after 6 and 12 months or at any time if a transformation to acute myeloid leukemia (AML) was detected. Applying a probe-panel targeting the centromere of chromosomes 7 and 8, 5q31, 5p15.2 and 7q31, we observed one case with 5q deletion not identified by G-banding. I-FISH at 6 and 12 months confirmed the karyotype results. Eight cases transformed to AML during follow-up, but no hidden clone was detected by I-FISH in any of them. The inclusion of I-FISH during follow-up of MDS resulted in a small improvement in abnormality detection when compared with conventional G-banding.
\end{abstract}

Key words: Myelodysplastic syndrome; FISH; G-banding

\section{Introduction}

Myelodysplastic syndrome (MDS) is a clonal hematopoietic stem cell disorder characterized by peripheral cytopenias due to ineffective hematopoiesis. Up to $30 \%$ of MDS patients are at risk to transform to acute leukemia (1). The identification of chromosomal abnormalities is crucial to determine survival and predict the risk of a transformation to acute leukemia (2). Nevertheless, a large proportion of MDS patients (40 to 65\%) (3) present normal karyotypes at diagnosis. In this group, which is highly heterogeneous from a biological standpoint, outcome is often unpredictable.

MDS is usually diagnosed with cytogenetic techniques such as G-banding. Some investigators have stated that the frequency of chromosomal abnormalities is underestimated with conventional techniques such as G-banding because the aberrant clone does not proliferate well in cell cultures, reducing the possibility of detection (3-5). It has also been proposed that hidden clones not detected by conventional cytogenetics might be involved in the progression to acute leukemia of MDS cases with a normal karyotype $(6,7)$, and that other, more sensitive approaches such as interphase fluorescent in situ hybridization (I-FISH) would be required for detection of occult lesions.

The purpose of the present study was to determine whether I-FISH would be able to detect hidden clones associated with the progression of MDS to acute myeloid leukemia (AML) that are undetected by conventional Gbanding methods over a 12-month period.

The present study was carried out within the context of a wide prospective study evaluating karyotype, FMS-like tyrosine kinase 3 (FLT3) internal tandem duplications, the French-American-British (FAB) Cooperative Group classification, the World Health Organization (WHO) classification of neoplastic diseases of the hematopoietic and lymphoid tissue, and the International Prognostic Scoring System (IPSS). I-FISH and G-banding were performed in 50 MDS patients at diagnosis and after 6 and 12 months, or whenever AML transformation was detected (8-13).

\section{Patients and Methods}

The group included 48 patients with primary MDS and 2 with secondary MDS diagnosed from March 2003 to March

Correspondence: M.L.L.F. Chauffaille, Disciplina de Hematologia e Hemoterapia, EPM, UNIFESP, Rua Botucatu, 740, $3^{\circ}$ andar, 04023-900 São Paulo, SP, Brasil. Fax: +55-11-5571-8806. E-mail: chauffaill@hemato.epm.br

Received March 28, 2009. Accepted September 25, 2009. Available online October 26, 2009. 
2006. Patients with de novo and secondary MDS were diagnosed according to WHO/FAB criteria $(14,15)$ and classified according to the IPSS (2). There were 27 (54\%) male and 23 $(46 \%)$ female patients. Treatment was based on red blood cell and platelet transfusions or bone marrow transplantation whenever appropriate. Median follow-up was 19 months (range: 2 to 36 months). Information concerning 8 of these 50 patients has been previously published (8-13). The study was approved by the Institution's Ethics Committee and all patients signed an informed consent form.

G-banding analysis was performed at the time of collection following standard protocols (16) and ISCN 2005 (17). Whenever possible, at least 20 metaphases were analyzed. I-FISH was performed on fixed bone marrow cell suspensions (5) that had been used for karyotyping and stored in Carnoy's fixative at $-20^{\circ} \mathrm{C}$. Since the most common chromosomal abnormalities in MDS involve chromosomes 5,7 and 8 , we used probes to detect deletions in these chromosomes. The following panel of probes was used: CEP 8 (Vysis, Zeis, USA), targeting the centromeric region of chromosome 8; LSI EGR1 Spectrum orange/:D5S721, LSI D5S23 Spectrum Green (Vysis), targeting 5q31 and 5p15.2, respectively, and Spectrum red D7S486, Spectrum green D7Z1, targeting 7q31 and 7 centromere (Vysis). The slides were prepared and incubated in $70 \%$ formamide/2X SSC at $73^{\circ} \mathrm{C}$ for $5 \mathrm{~min}$, dehydrated in ethanol $(70,85$ and $100 \%)$ and air-dried. I-FISH assays were performed following probe manufacturer instructions. The slides were analyzed using an epifluorescence microscope (Zeiss, Switzerland) with a triple band pass filter and single filters for FITC, Texas red and DAPI (Power Gene - Image Analysis Software, Applied Imaging Corporation, USA).

A minimum of 200 cells per specimen/probe were scored by two independent investigators. In the absence of discrepancy the results were reviewed, combined and averaged (5). FISH and G-banding were analyzed blindly regarding each other. Samples from 9 bone marrow donors (without MDS) were used as controls. Based on the rate of abnormalities observed in controls plus two standard deviations, the cut-off level for normal values was established for each target region (5) as follows: $-5=3 \%$, del $(5 q 31)=$ $3 \%,-7=1.5 \%$, del $(7 q 31)=0.6 \%,+8=2.7 \%$.

\section{Results}

In terms of IPSS, 13 patients were classified as low-risk, 27 as intermediate 1, 3 as intermediate 2, and 2 as highrisk for progression to AML. Evaluation was not possible in 4 patients.

G-banding revealed chromosomal abnormalities in 9 of 44 patients $(20.4 \%)$ at diagnosis, 9 of 36 patients $(25 \%)$ at 6 months and 9 of 29 patients (31\%) at 12 months. Sufficient metaphases for analysis were not obtained in 6 cases at diagnosis, 5 cases at 6 months and 3 cases at 12 months. I-FISH revealed abnormalities in 6 of 50 patients $(12 \%)$ at diagnosis, confirming G-banding findings in 5 cases. In one case with insufficient metaphases on G-banding, I-FISH revealed deletion of $5 q$. I-FISH results at 6 and 12 months confirmed the karyotyping.

During follow-up, the diagnosis of 8 patients became AML. Five patients presented chromosomal abnormalities at the time of transformation. The FISH panel did not detect hidden clones related to $\mathrm{AML}$ transformation. Ten patients died during the 12-month follow-up period due to severe cytopenia or to no response to chemotherapy (after AML transformation).

\section{Discussion}

To the best of our knowledge, this is the first study to compare the detection of chromosomal abnormalities with the I-FISH and G-banding methods over a 12-month follow-up period in patients with MDS. I-FISH revealed an additional abnormality at diagnosis in only 1 patient $(2 \%)$, i.e., deletion $5 q$ in 1 patient who presented no metaphases in karyotype analysis. Notwithstanding its low additional benefit, FISH was important to define the best treatment for this specific patient, since lenalidomide (Revlimid ${ }^{\circledR}$, Cellgene, USA) induces a complete hematological and cytogenetic response in more than $80 \%$ of MDS patients with $5 q$ deletion (18).

Several studies have compared FISH and conventional cytogenetic analysis at specific times during the development of the diseases, most of them showing only a small advantage of FISH for detecting chromosomal anomalies. Romeo et al. (5) evaluated 40 MDS patients at diagnosis by G-banding karyotype and dual-color FISH and reported that FISH was able to detect an abnormal clone in 3 of 22 patients with a normal karyotype and in 1 of 6 patients with insufficient metaphases. In a study by the Eastern Cooperative Oncology Group (ECOG), only 1 of 48 patients presented an occult deletion of chromosome 11 detected by FISH (6). Panani and Pappa (19) studied the incidence of trisomy 8 in 30 primary MDS patients using FISH and G-banding. Superiority of FISH over karyotyping was detected in only $3(10 \%)$ cases. Recently, Chen et al. (20) observed that I-FISH, but not G-banding, was able to detect $-5 / 5 q$ - in 5 of 30 cases, $-7 / 7 q$ - in 3 cases, and trisomy 8 in 5 cases.

Rigolin et al. (3) analyzed 101 patients with primary MDS and a normal karyotype and observed $15-32 \%$ of interphase cells with at least one FISH abnormality. In that study, FISH abnormalities were associated with a higher rate of progression to AML (13/18 vs 12/83) and were predictive of a worse prognosis. It should be noted that the patients in the study by Rigolin et al. (3) were mostly in the intermediate 2 or high-risk IPSS categories. This might explain why an increased number of FISH abnormalities or AML evolution was detected in relation to the present study, which included mostly low-risk and intermediate 1 patients.

The use of I-FISH during follow-up of MDS did not im- 
prove the detection of abnormalities when compared with conventional G-banding. I-FISH should probably be used in selected cases for which specific therapeutic options must be considered.

\section{References}

1. Mufti GJ. Pathobiology, classification, and diagnosis of myelodysplastic syndrome. Best Pract Res Clin Haematol 2004; 17: 543-557.

2. Greenberg P, Cox C, LeBeau MM, Fenaux P, Morel P, Sanz G, et al. International scoring system for evaluating prognosis in myelodysplastic syndromes. Blood 1997; 89: 2079-2088.

3. Rigolin GM, Bigoni R, Milani R, Cavazzini F, Roberti MG, Bardi $A$, et al. Clinical importance of interphase cytogenetics detecting occult chromosome lesions in myelodysplastic syndromes with normal karyotype. Leukemia 2001; 15: 1841-1847.

4. Sole F, Luno E, Sanzo C, Espinet B, Sanz GF, Cervera J, et al. Identification of novel cytogenetic markers with prognostic significance in a series of 968 patients with primary myelodysplastic syndromes. Haematologica 2005; 90: 11681178.

5. Romeo M, Chauffaille ML, Silva MR, Bahia DM, Kerbauy J. Comparison of cytogenetics with FISH in 40 myelodysplastic syndrome patients. Leuk Res 2002; 26: 993-996.

6. Cherry AM, Brockman SR, Paternoster SF, Hicks GA, Neuberg D, Higgins RR, et al. Comparison of interphase FISH and metaphase cytogenetics to study myelodysplastic syndrome: an Eastern Cooperative Oncology Group (ECOG) study. Leuk Res 2003; 27: 1085-1090.

7. Trost D, Hildebrandt B, Muller N, Germing U, Royer-Pokora B. Hidden chromosomal aberrations are rare in primary myelodysplastic syndromes with evolution to acute myeloid leukaemia and normal cytogenetics. Leuk Res 2004; 28 : 171-177.

8. Feitosa Pinheiro R, Maranhao Bahia D, Artur Flores Pelloso L, Guimaraes Vaz De Campos M, De Lourdes Chauffaille M. Isolated interstitial $9 q$ deletion in a case of unclassifiable myelodysplastic syndrome. Cancer Genet Cytogenet 2004; 153: 183-184.

9. Pinheiro RF, Silva MR, Chauffaille ML. The $5 q-$ syndrome and autoimmune phenomena: report of three cases. Leuk Res 2006; 30: 507-510.

10. Pinheiro RF, Chauffaille ML, Silva MR. A rare case of acute myeloid leukemia evolving from a myelodysplastic syndrome

\section{Acknowledgments}

This research was supported by FAPESP (\#04/2009-6). M.L.L. Chauffaille is the recipient of CNPq grants.

with der(19)t(1;19). Cancer Genet Cytogenet 2006; 169: 181-183.

11. Pinheiro RF, Chauffaille ML, Silva MR. Isochromosome 17q in MDS: a marker of a distinct entity. Cancer Genet Cytogenet 2006; 166: 189-190.

12. Pinheiro RF, Moreira Ede S, Silva MR, Greggio B, Alberto FL, Chauffaille Mde L. FLT3 mutation and AML/ETO in a case of myelodysplastic syndrome in transformation corroborates the two hit model of leukemogenesis. Leuk Res 2007; 31: 1015-1018.

13. Pinheiro RF, de Sa Moreira E, Silva MR, Alberto FL, Chauffaille Mde L. FLT3 internal tandem duplication during myelodysplastic syndrome follow-up: a marker of transformation to acute myeloid leukemia. Cancer Genet Cytogenet 2008; 183: 89-93.

14. Harris NL, Jaffe ES, Diebold J, Flandrin G, Muller-Hermelink HK, Vardiman J, et al. World Health Organization classification of neoplastic diseases of the hematopoietic and lymphoid tissues: report of the Clinical Advisory Committee Meeting-Airlie House, Virginia, November 1997. J Clin Oncol 1999; 17: 3835-3849.

15. Bennett JM, Catovsky D, Daniel MT, Flandrin G, Galton DA, Gralnick HR, et al. Proposals for the classification of the myelodysplastic syndromes. Br J Haematol 1982; 51: 189199.

16. Chauffaille ML, Yamamoto M, Moncau JE, Braga GW, Souto EX, Kerbauy J. [Cytogenetic abnormalities as prognostic factors in acute myeloid leukemia]. Rev Assoc Med Bras 1996; 42: 200-204.

17. Mitelman F. Catalog of chromosome aberrations in cancer. 4th edn. New York: Wiley-Liss; 2005.

18. List AF, Baker AF, Green S, Bellamy W. Lenalidomide: targeted anemia therapy for myelodysplastic syndromes. Cancer Control 2006; 13 (Suppl): 4-11.

19. Panani AD, Pappa V. Hidden chromosome 8 abnormalities detected by FISH in adult primary myelodysplastic syndromes. In Vivo 2005; 19: 979-981.

20. Chen L, Li J, Zhu Y, Qiu H, Pan J, Wang R, et al. Conventional and molecular cytogenetic features of myelodysplastic syndrome in China. Exp Oncol 2007; 29: 299-303. 